\title{
A pesquisa sobre envelhecimento humano no Brasil: pesquisadores, temas e tendências
}

\author{
The research about human aging in Brazil: \\ researchers, themes, and tendencies
}

Shirley Donizete Prado 1 Jane Dutra Sayd 1

\footnotetext{
1 Universidade do Estado do Rio de Janeiro (UERJ), na Universidade Aberta da Terceira Idade (UnATI), Gerência de Pesquisa, Centro de Documentação sobre Envelhecimento (CRDE).

Rua São Francisco Xavier, 524/10o andar, Bloco F, Pavilhão João Lyra Filho, Maracanã, 20559-900, Rio de Janeiro RJ. prados@uerj.br
}

Abstract We discuss investigated themes and tendencies of the research about human aging in Brazil from indicators that relate the degrees of the researchers and students inserted in the research groups that constitute the Executive Committee of the Research Groups in Brazil (2002) of the Scientifical and Technological Development National Council (CNPq) in its 4.1 version. The criterion to identify the studied groups was the one of, at least, the development of a research line related to the human aging and the search in the data base was done from key-words related to the human aging process. There were 144 groups identified, 209 research lines, and 511 researchers. The results suggest a reduced power of reproduction of this labour force, even lesser among the groups that focus on, exclusively, the human aging study. They also show that the route in the direction to the research scientific consolidation to these particular groups will be much longer than the one to other areas, such as, epidemiology.

Key words Aging, Aged, Research, Tendencies, Researchers, Scientific production, Brazil
Resumo Discutimos temas investigados e tendências da pesquisa sobre envelhecimento humano no Brasil a partir de indicadores que relacionam a titulação de pesquisadores e estudantes inseridos nos grupos de pesquisa que compõem o Diretório dos Grupos de Pesquisa no Brasil (2002) do Conselho Nacional de Desenvolvimento Científico e Tecnológico ( $\mathrm{CNPq}$ ) em sua versão 4.1. O critério para a identificação dos grupos estudados foi o desenvolvimento de, pelo menos, uma linha de pesquisa referente ao envelhecimento humano, e a busca na base de dados foi realizada a partir de palavras-chave associadas ao processo de envelhecimento humano. Foram identificados 144 grupos, 209 linhas de pesquisa e 511 pesquisadores. Os resultados sugerem uma reduzida capacidade de reprodução desta força de trabalho, menor ainda entre os grupos que se voltam exclusivamente para o estudo do envelhecimento humano. Indicam também que o percurso em direção à consolidação científica da pesquisa para esses grupos específicos há de ser bem mais longo que para outras áreas, como a epidemiologia, por exemplo.

Palavras-chave Envelhecimento, Idoso, Pesquisa, Tendências, Pesquisadores, Produção científica, Brasil 


\section{Introdução}

O presente trabalho dá continuidade ao artigo intitulado "A pesquisa sobre envelhecimento humano no Brasil: grupos e linhas de pesquisa" (Prado, 2003), no qual discutimos as áreas de conhecimento em que se inserem os grupos de pesquisa que contam com pelo menos uma linha de pesquisa relativa ao envelhecimento, o período de seu surgimento e sua distribuição geográfica e institucional. Tomamos, então, como referência central, o estudo de Guimarães et al. (2001), que analisa a pesquisa em epidemiologia no Brasil.

Esses autores também nos conduzem a uma importante reflexão acerca dos pesquisadores, estudantes, suas respectivas titulações e publicações, problematizando, a partir da construção de indicadores para avaliação de tendências em pesquisa científica, a capacidade de reprodução da força de trabalho e a consolidação da epidemiologia como campo de produção de conhecimento. Esta última abordagem também aqui nos orienta nas discussões sobre o dinamismo e a consolidação da pesquisa voltada para os idosos, a velhice e demais aspectos do processo de envelhecimento humano no Brasil.

\section{Procedimentos metodológicos}

Construímos uma estratégia de busca no Diretório dos Grupos de Pesquisa no Brasil, em sua versão 4.1, desenvolvido pelo Conselho Nacional de Desenvolvimento Científico e Tecnológico (CNPq, 2002). Utilizamos palavras-chave associadas ao envelhecimento humano e pesquisamos nos seguintes campos do Diretório: Nome do grupo, Nome da linha de pesquisa e Palavraschave da linha de pesquisa. Este procedimento, realizado em julho de 2001, possibilitou a identificação de 144 grupos com 209 linhas de pesquisa descritas pelos líderes como ativas na geração de conhecimento relativo ao envelhecimento humano no Brasil. Descrição mais detalhada dos procedimentos metodológicos encontra-se em artigo que aborda os grupos e linhas de pesquisa voltados para essa temática (Prado, 2004).

No interior desses grupos foram identificados dois subconjuntos: 43 grupos especificos, ou seja, aqueles que se voltam exclusivamente para o estudo dos idosos, da velhice e do processo de envelhecimento e 101 grupos não específicos, ou seja, que têm a temática em tela como uma questão abordada em uma área maior de interesse.

\section{Pesquisadores}

Os 144 grupos que compõem este estudo incluem 610 linhas de pesquisa e são compostos por 931 pesquisadores, observando que há repetição de nomes, uma vez que ocorre a participação do mesmo pesquisador em mais de um grupo de pesquisa.

Nos 43 grupos específicos identificamos 192 pesquisadores atuando em 92 linhas de pesquisa para a geração de conhecimento sobre envelhecimento humano (já descontadas repetições de pesquisadores que atuam em mais de um grupo ou linha de pesquisa).

Quanto aos outros 101 grupos não específi$\cos$, das 608 linhas de pesquisa desenvolvidas em seu interior, 117 estão voltadas para objetos relativos ao envelhecimento humano e destas participam 331 pesquisadores.

Além da simultânea participação de pesquisadores em mais de uma linha ou grupo de pesquisa, é necessário registrar que o aparecimento do nome de um determinado pesquisador na lista dos membros de uma linha de pesquisa que pertence a um grupo não especifico não é suficiente para garantir que ele efetivamente participe de estudos sobre envelhecimento. Com freqüência, uma mesma linha de pesquisa se refere a vários temas, entre os quais, algum relativo ao envelhecimento. Um exemplo esclarecedor corresponde a uma linha de pesquisa voltada para avaliação de programas de saúde para mulher, criança, adolescente, adulto e idoso e que conta com vários pesquisadores; não é possível saber se apenas alguns dos pesquisadores desta linha se dedicam ao estudo de um determinado programa ou se todos trabalham com todos os programas. Assim, não é possível saber quantos e/ou quais pesquisadores estão trabalhando com avaliação de programas para idosos. Por conta desta característica dos dados do Diretório, acreditamos que o número de pesquisadores esteja superestimado, mas neste momento não temos meios de avaliar em que grau isto acontece.

Por outro lado, certamente há grupos que trabalham com temas relativos ao envelhecimento e que não aparecem neste estudo por não terem registrado palavras-chave associadas a este processo específico e sim às patologias ou processos aos quais se dedicam; poderia ser, por exemplo, o caso de pesquisadores que estudam doenças crônicas e degenerativas, altamente associadas à velhice, ao processo de envelhecimento humano. Por esse ângulo, nossos 
números estariam subestimados e, da mesma forma, não temos ainda parâmetros para dimensionar esse acontecimento.

Assim, partindo dos dados disponíveis no Diretório de Grupos de Pesquisa, podemos afirmar que 511 pesquisadores participam de 209 linhas de pesquisa que fazem referência ao envelhecimento humano compondo 144 grupos de pesquisa. Observemos que não é possível somar os 192 pesquisadores dos grupos especificos com os 331 pesquisadores dos grupos não específicos, porque há repetição entre esses dois subconjuntos. Uma vez descontadas todas as situações em que um mesmo pesquisador participa de mais de um grupo ou linha de pesquisa, chegamos a 511 pesquisadores no total. Esta é a melhor aproximação que podemos fazer a respeito da massa crítica a gerar conhecimento sobre envelhecimento humano no País.

Os resultados mostram que 172 pesquisadores são líderes, já descontadas as situações em que o mesmo pesquisador é líder de mais de um grupo, sendo que 61 pesquisadores mantêm a condição de liderança de grupos específicos e 111 de grupos não específicos. Também cabem aqui as mesmas ressalvas acerca da duplicação de dados registrada nos parágrafos anteriores.

A tabela 1 nos mostra que $55,6 \%$ dos 511 pesquisadores que participam de linhas de pesquisa sobre envelhecimento apresentam doutorado como titulação máxima, proporção semelhante àquela presente em toda a base de dados do Diretório (56,7\%). Já a proporção de líderes com doutorado (79\%) nos grupos que contam com linhas de pesquisa relativas ao en- velhecimento se apresenta aquém da registrada para o conjunto dos pesquisadores brasileiros, que é de $83,5 \%$. Mas, observando em perspectiva mais detalhada, temos que entre os líderes dos grupos não específicos $85,0 \%$ são doutores, enquanto entre os grupos específicos apenas $72,9 \%$ alcançaram este grau. Mais uma vez encontramos indicações de que as pesquisas sobre envelhecimento estão marcadas, ao menos em parte, por um caráter inicial, com a existência de grupos liderados por pesquisadores que ainda têm um percurso de formação acadêmica a seguir.

Foi possível alcançar os curriculum vitae Lattes-CNPq (CNPq, 2002a) para 160 dos 284 pesquisadores doutores. Assim como no caso da epidemiologia, a maioria obteve o título de doutor a partir de 1990, conforme consta da tabela 2 .

Uma quinta parte dos pesquisadores declarou ter obtido o título doutoral no exterior, o que está aquém do que se passa com o conjunto do Diretório e com a epidemiologia, que contam com uma terça parte de seus pesquisadores com doutorado no exterior. A tabela 3 nos mostra que os países da Europa têm peso importante na formação dos pesquisadores do envelhecimento; o Japão aparece aqui, certamente, por conta de sua projeção internacional no campo dos estudos sobre envelhecimento e da cooperação interinstitucional estabelecida entre o Instituto de Geriatria e Gerontologia da PUC-RS e universidades japonesas. Para o conjunto do Diretório, os Estados Unidos respondem pela liderança na formação de doutores.

Tabela 1

Pesquisadores que atuam nas linhas de pesquisa referentes ao envelhecimento humano por titulação segundo liderança. Brasil, 2000.

\begin{tabular}{|c|c|c|c|c|c|c|}
\hline \multirow[t]{3}{*}{ Titulação } & \multicolumn{6}{|c|}{ Pesquisadores } \\
\hline & \multicolumn{2}{|c|}{ Líderes 1} & \multicolumn{2}{|c|}{ Não líderes 2} & \multicolumn{2}{|c|}{ Total 3} \\
\hline & $\mathrm{N}$ & $\%$ & $\mathrm{~N}$ & $\%$ & $\mathrm{~N}$ & $\%$ \\
\hline Doutorado & 136 & 79,0 & 157 & 45,0 & 284 & 55,6 \\
\hline Mestrado & 28 & 16,3 & 121 & 34,7 & 149 & 29,2 \\
\hline Atualização/Especialização & 7 & 4,1 & 56 & 16,0 & 63 & 12,3 \\
\hline Graduação & 1 & 0,6 & 15 & 4,3 & 15 & 2,9 \\
\hline Total & 172 & 100,0 & 349 & 100,0 & 511 & 100,0 \\
\hline
\end{tabular}

Fonte: CNPq - Diretório dos Grupos de Pesquisa no Brasil, versão 4.1, 2002.

1 Um pesquisador lidera quatro grupos e cinco pesquisadores lideram dois grupos cada.

2 Um pesquisador participa de três grupos e 15 pesquisadores participam de dois grupos cada.

3 Um pesquisador atua em quatro grupos, quatro pesquisadores atuam em três grupos cada

e 25 pesquisadores participam de dois grupos simultaneamente. 


\section{Tabela 2}

Pesquisadores que atuam em linhas de pesquisa referentes ao envelhecimento humano e que obtiveram título de doutor segundo década de doutoramento. Brasil, 2000.

\begin{tabular}{lrr}
\hline Década de doutoramento & \multicolumn{2}{c}{ Pesquisadores } \\
& N & $\%$ \\
\hline $1970-1979$ & 9 & 5,7 \\
$1980-1989$ & 29 & 18,5 \\
$1990-1999$ & 103 & 65,6 \\
$2000-2002$ & 16 & 10,2 \\
Total & $\mathbf{1 5 7 ^ { \star }}$ & $\mathbf{1 0 0 , 0}$ \\
\hline
\end{tabular}

Fonte: $\mathrm{CNPq}$ - Diretório dos Grupos de Pesquisa no Brasil, versão 4.1, e CV Lattes-CNPq, 2002.

* Em três currículos não havia informação sobre o ano do doutoramento.

\begin{tabular}{|c|c|c|}
\hline $\begin{array}{l}\text { Tabela } 3 \\
\text { Pesquisadores que atuam em linhas de } \\
\text { ao envelhecimento humano e que obti } \\
\text { no exterior segundo país. Brasil, } 2000 .\end{array}$ & $\begin{array}{l}\text { iisa re } \\
\text { títulc }\end{array}$ & entes \\
\hline País de obtenção do título de doutor & Pesq & adores \\
\hline & $\mathrm{N}$ & $\%$ \\
\hline Grã-Bretanha & 6 & 17,6 \\
\hline França & 5 & 14,7 \\
\hline Alemanha & 4 & 11,8 \\
\hline Espanha & 4 & 11,8 \\
\hline Estados Unidos & 4 & 11,8 \\
\hline Japão & 4 & 11,8 \\
\hline Bélgica & 3 & 8,8 \\
\hline Canadá & 2 & 5,9 \\
\hline Itália & 1 & 2,9 \\
\hline México & 1 & 2,9 \\
\hline Total & 34 & 100,0 \\
\hline
\end{tabular}

Fonte: $\mathrm{CNPq}$ - Diretório dos Grupos de Pesquisa no Brasil, versão 4.1, 2002 e CV Lattes-CNPq, 2002.

A predominância do Estado de São Paulo e, em particular, da USP na titulação doutoral dos pesquisadores no Brasil é coerente com o perfil do Diretório e da epidemiologia. O Rio de Janeiro e o Rio Grande do Sul também têm papel importante na titulação desses pesquisadores, este último provavelmente como repercussão do Programa de Pós-Graduação em Gerontologia Biomédica da PUC-RS. A grande diversidade das áreas de conhecimento envolvidas na pesquisa sobre envelhecimento certamente é o elemento explicativo para as similaridades encontradas com o perfil do Diretório em vários dados abordados neste estudo. A ta- bela 4 nos apresenta os dados referentes às instituições onde os pesquisadores concluíram o doutorado e a tabela 5 as unidades da federação dessas instituições.

\section{Estudantes}

O estudantes aparecem nos grupos sem que seja possível distinguir as linhas de pesquisa às quais estão ligados, como procedemos em relação aos pesquisadores. Assim, nos grupos não específicos estão juntos estudantes que atuam tanto nas linhas que tratam do envelhecimento humano quanto nas que estão voltadas para outros temas. A tabela 6 nos mostra que a proporção de doutorandos nos grupos específicos (10,4\%) é bem menor que entre os grupos não específicos $(15,2 \%)$ e que no campo da epidemiologia (17,9\%).

Indicadores de "dinamismo" e "consolidação científica" para algumas grandes áreas, áreas e para a epidemiologia no Brasil são apresentados na tabela 7. Guimarães et al. (2001) reafirmam a idéia de que é do conjunto dos doutorandos que surgirão os novos pesquisadores de uma determinada área e que a relação entre o número de doutorandos e pesquisadores doutores pode expressar a "intensidade de reprodução da força de trabalho em pesquisa" ou seu dinamismo. Além disso, os autores entendem que a consolidação científica de uma área pode ser inferida a partir da relação entre o número de doutores e o conjunto de estudantes em todos os graus de qualificação. Assim, concluem que a epidemiologia apresenta um alto dinamismo, mas ainda tem um longo percurso até que venha a se colocar em condição de igualdade com as áreas mais consolidadas de pesquisa no País.

Estes mesmos indicadores construídos para os grupos que contam com linhas de pesquisa relativas ao envelhecimento humano estão apresentadas na tabela 8 . Os resultados sugerem baixo dinamismo, menor ainda nos grupos específicos de pesquisa relativa ao envelhecimento humano no País. Indicam também que o percurso em direção à consolidação científica da pesquisa sobre envelhecimento há de ser bem mais longo que o da epidemiologia, especialmente para os grupos específicos. 
Tabela 4

Pesquisadores que atuam em linhas de pesquisa referentes ao envelhecimento humano e que obtiveram título de doutor no Brasil segundo instituição. Brasil, 2000.

\begin{tabular}{lrc}
\hline Instituição & \multicolumn{2}{c}{ Pesquisadores } \\
& $\mathrm{N}$ & $\%$ \\
\hline USP - Universidade de São Paulo & 53 & 42,1 \\
UFRJ - Universidade Federal do Rio de Janeiro & 14 & 11,1 \\
Unicamp - Universidade Estadual de Campinas & 9 & 7,1 \\
PUCRS - Pontifícia Universidade Católica do Rio Grande do Sul & 6 & 4,8 \\
UFRGS - Universidade Federal do Rio Grande do Sul & 6 & 4,8 \\
Unesp - Universidade Estadual Paulista Júlio de Mesquita Filho & 6 & 4,8 \\
Unifesp - Universidade Federal de São Paulo & 6 & 4,8 \\
UFMG - Universidade Federal de Minas Gerais & 5 & 4,0 \\
UERJ - Universidade do Estado do Rio de Janeiro & 2 & 1,6 \\
UFPE - Universidade Federal de Pernambuco & 2 & 1,6 \\
Fiocruz - Fundação Oswaldo Cruz & 2 & 1,6 \\
PUCSP - Pontifícia Universidade Católica de São Paulo & 2 & 1,6 \\
UFSC - Universidade Federal de Santa Catarina & 2 & 1,6 \\
UFPR - Universidade Federal do Paraná & 2 & 1,6 \\
UPE - Universidade de Pernambuco & 2 & 1,6 \\
UFBA - Universidade Federal da Bahia & 1 & 0,8 \\
UnB - Universidade de Brasília & 1 & 0,8 \\
UFPB - Universidade Federal da Paraíba & 1 & 0,8 \\
UFSCAR - Universidade Federal de São Carlos & 1 & 0,8 \\
Unimep - Universidade Metodista de Piracicaba & 1 & 0,8 \\
UFRRJ - Universidade Federal Rural do Rio de Janeiro & 1 & 0,8 \\
UFSM - Universidade Federal de Santa Maria & 1 & 0,8 \\
Total & $\mathbf{1 2 6}$ & $\mathbf{1 0 0 , 0}$ \\
\hline & &
\end{tabular}

Fonte: CNPq - Diretório dos Grupos de Pesquisa no Brasil, versão 4.1, 2002 e CV Lattes-CNPq, 2002.

\section{Produção científica}

Para abordar a produção científica dos grupos que contam com linhas de pesquisa sobre envelhecimento, tomamos os dados disponíveis na versão 4.1 do Diretório que correspondem ao conteúdo do curriculum vitae Lattes de cada pesquisador para o período de 1997 a 2000. É importante ressalvar que esta produção científica não se refere exclusivamente ao envelhecimento humano uma vez que parcela significativa dos pesquisadores trata também de outros objetos. Os dados estão superestimados também por conta da múltipla contagem, ou seja, quando um mesmo trabalho tem co-autores e estes atuam em mais de uma linha ou grupo de pesquisa, o trabalho será contado para cada autor em cada linha ou grupo. Nesta versão 4.1, ainda não havia obrigatoriedade de implantação do CV Lattes para os pesquisadores e estudantes inseridos nos grupos, de forma que nem toda produção encontra-se aí registrada.
Tabela 5

Pesquisadores que atuam em linhas de pesquisa referentes ao envelhecimento humano e que obtiveram título de doutor no Brasil segundo Estado. Brasil, 2000.

\begin{tabular}{lrr}
\hline \multirow{2}{*}{ Estado } & \multicolumn{2}{c}{ Pesquisadores } \\
& $\mathrm{N}$ & $\%$ \\
\hline São Paulo & 78 & 61,9 \\
Rio de Janeiro & 19 & 15,1 \\
Rio Grande do Sul & 13 & 10,3 \\
Minas Gerais & 5 & 4,0 \\
Pernambuco & 4 & 3,2 \\
Santa Catarina & 2 & 1,6 \\
Paraná & 2 & 1,6 \\
Bahia & 1 & 0,8 \\
Distrito Federal & 1 & 0,8 \\
Paraíba & 1 & 0,8 \\
Total & $\mathbf{1 2 6}$ & $\mathbf{1 0 0 , 0}$
\end{tabular}

Fonte: $\mathrm{CNPq}$ - Diretório dos Grupos de Pesquisa no Brasil, versão 4.1, 2002 e CV Lattes-CNPq, 2002. 
Tabela 6

Estudantes orientados por pesquisadores dos grupos de pesquisa que contam com linhas de pesquisa referentes ao envelhecimento humano por nível de treinamento. Brasil, 2000.

\begin{tabular}{lrrrrrr}
\hline Nível de treinamento & \multicolumn{4}{c}{ Estudantes } & \multicolumn{2}{c}{ Total } \\
& N & \multicolumn{2}{c}{ \% } & \multicolumn{2}{c}{ Grupos não específicos } & \multicolumn{2}{c}{ N } & $\%$ \\
\hline Doutorado & 19 & 10,4 & 122 & 15,2 & 141 & 14,3 \\
Mestrado & 79 & 43,4 & 300 & 37,3 & 379 & 38,4 \\
Especialização & 11 & 6,0 & 39 & 4,8 & 50 & 5,1 \\
Graduação & 73 & 40,1 & 344 & 42,7 & 417 & 42,2 \\
Total & $\mathbf{1 8 2}$ & $\mathbf{1 0 0 , 0}$ & $\mathbf{8 0 5}$ & $\mathbf{1 0 0 , 0}$ & $\mathbf{9 8 7}$ & $\mathbf{1 0 0 , 0}$ \\
\hline
\end{tabular}

Fonte: CNPq - Diretório dos Grupos de Pesquisa no Brasil, versão 4.1, 2002.

\section{Tabela 7}

Relações entre estudantes e pesquisadores segundo algumas grandes áreas, áreas e na pesquisa em epidemiologia. Brasil, 2000.

\begin{tabular}{lcc}
\hline Áreas & $\begin{array}{c}\text { Doutorandos/ } \\
\text { Pesquisadores doutores }\end{array}$ & $\begin{array}{c}\text { Doutorandos/Total de } \\
\text { estudantes nos grupos }\end{array}$ \\
\hline Ciências Exatas e da Terra & 0,47 & 0,30 \\
Ciências Biológicas & 0,50 & 0,22 \\
Ciências Humanas & 0,36 & 0,17 \\
Ciências Sociais Aplicadas & 0,19 & 0,10 \\
Ciências da Saúde & 0,34 & 0,19 \\
Saúde Coletiva & 0,32 & 0,17 \\
Epidemiologia & 0,40 & 0,18 \\
Todas as áreas & 0,42 & 0,22 \\
\hline
\end{tabular}

Fonte: Guimarães et al., 2001.

\section{Tabela 8}

Relações entre estudantes e pesquisadores nos grupos de pesquisa que contam com linhas de pesquisa referentes ao envelhecimento humano. Brasil, 2000.

\begin{tabular}{lccc}
\hline Indicadores & \multicolumn{3}{c}{$\begin{array}{c}\text { Grupos de pesquisa com linha de pesquisa } \\
\text { referente ao envelhecimento humano }\end{array}$} \\
& Específicos & Não específicos & Total \\
\hline Doutorandos/Pesquisadores doutores & 0,18 & 0,28 & 0,26 \\
Doutorandos/Total de estudantes nos grupos & 0,10 & 0,15 & 0,14 \\
\hline
\end{tabular}

Fonte: $\mathrm{CNPq}$ - Diretório dos Grupos de Pesquisa no Brasil, versão 4.1, 2002. 
A exemplo da epidemiologia, tomamos alguns itens da produção bibliográfica e atividades de orientação de teses e dissertações sobre envelhecimento, que se encontram apresentados na tabela 9, distribuídos por algumas grandes áreas do conhecimento. Predominam os artigos de circulação nacional entre os grupos inseridos na grande área das Ciências da Saúde, o que pode estar ligado, ao menos em algum grau, a interesses de cunho nacional, como políticas, intervenções entre outros. Nas Ciências Biológicas, destacam-se os artigos de circulação internacional, o que mantém coerência com a trajetória da ciência básica, sempre mais afeita às discussões no plano mundial. Nas Humanidades, artigos de circulação nacional, livros e capítulos de livros são veículos preferidos. Esses resultados mostram que os padrões que caracterizam as formas privilegiadas para divulgação da produção de conhecimento nas diferentes áreas estão presentes também para o campo do envelhecimento, conforme discussões presentes em Guimarães et al. (2001).

\section{Temas estudados}

Para a identificação dos temas pesquisados sobre velhos, velhice e envelhecimento, tentamos, inicialmente, trabalhar a partir de listas de temas adotadas em congressos nacionais de geriatria e gerontologia para classificação de trabalhos apresentados. Este procedimento resultou infrutífero dada a alta especialização dos temas escolhidos. Partimos, então, para a construção de uma lista de temas a partir dos conteúdos do campo nome do grupo do Diretório. Uma vez estabelecida uma listagem preliminar, tomamos as 209 linhas de pesquisa e procedemos à classificação de cada em até quatro temas. As situações de dúvida foram dirimidas por meio da leitura dos demais campos disponibilizados no Diretório: Nome do grupo, Nome da linha de pesquisa, Palavras-chave da linha de pesquisa e Repercussões do grupo. Ao final desta segunda etapa do processo de classificação, alguns temas foram incluídos e outros foram aprimorados em função das informações presentes nos campos considerados.

Esta foi uma das tarefas mais difíceis que enfrentamos no desenvolvimento deste trabalho. O processo de construção desta lista de temas exigiu inúmeras leituras dos dados presentes nos formulários do Diretório, muitas vezes sintéticos demais para nos fornecerem infor- mações conclusivas. Assim, o resultado final é antes de tudo provisório, posto que é uma listagem arbitrada pelos pesquisadores envolvidos no processo de sua construção. Certamente que a análise da produção científica brasileira sobre envelhecimento trará sua revisão com resultados bastante mais precisos.

A tabela 10 indica a predominância da pesquisa relativa às doenças crônicas e degenerativas e síndromes geriátricas. Como se trata de patologias que envolvem hábitos, comportamentos, estilos de vida, é razoável que o segundo tema mais pesquisado esteja situado no campo da educação, promoção da saúde e prevenção de doenças. O conhecimento do processo biológico de envelhecimento humano parece apresentar-se como uma das necessidades/prioridades mais cadentes para os cientistas.

O tema epidemiologia nos permite algumas comparações com padrões nacional e mundial de pesquisa. A epidemiologia da terceira idade foi mencionada em 19 das linhas da pesquisa epidemiológica brasileira, o que coincide com achados deste nosso estudo. Guimarães et al. (2001) apresentam, também, dados comparativos sobre a pesquisa em epidemiologia entre Brasil e Estados Unidos, onde é possível verificar que o tema epidemiologia da terceira idade corresponde a algo entre $2 \%$ a $3 \%$ do conjunto da pesquisa epidemiológica americana, valores próximos aos $4,7 \%$ que encontramos no interior das linhas de pesquisa brasileiras relativas ao envelhecimento humano. São resultados que indicam, por um lado, a consistência deste nosso trabalho e, por outro, que a pesquisa epidemiológica nacional relativa ao envelhecimento humano parece acompanhar o padrão mundial de geração de conhecimento neste campo.

Estes resultados parecem apresentar-se em sintonia com os temas identificados quando do desenvolvimento do Catálogo de teses e dissertações na área do envelhecimento (Prado et al., 1999). O amplo leque temático é coerente com o grande número de áreas do conhecimento encontradas na análise da base de dados sobre programas de pós-graduação com linhas de pesquisa relativas ao envelhecimento no Brasil (Prado e Lima, 2000)

\section{Considerações finais}

A pesquisa relativa à velhice, ao velho e ao processo de envelhecimento se volta de forma enfática para o estudo das doenças crônicas e dege- 
Tabela 9

Produção científica dos grupos que contam com linhas de pesquisa relativas ao envelhecimento humano por grandes áreas selecionadas no período 1997-2000. Brasil, 2001.

\begin{tabular}{|c|c|c|c|c|c|c|c|}
\hline $\begin{array}{l}\text { Grandes áreas } \\
\text { do conhecimento } \\
\text { selecionadas }\end{array}$ & $\begin{array}{c}\text { Artigo } \\
\text { circulação } \\
\text { nacional } \\
\text { N }\end{array}$ & $\begin{array}{c}\text { Artigo } \\
\text { circulação } \\
\text { internacional } \\
\mathrm{N}\end{array}$ & Livros & $\begin{array}{l}\text { Capítulos } \\
\text { de livros }\end{array}$ & Teses & Dissertações & Total \\
\hline Ciências da Saúde & 1.566 & 583 & 104 & 437 & 164 & 504 & 3.358 \\
\hline Ciências Biológicas & 265 & 542 & 26 & 120 & 77 & 170 & 1.200 \\
\hline Ciências Humanas & 309 & 37 & 73 & 206 & 47 & 208 & 880 \\
\hline Ciências Sociais Aplicadas & 112 & 23 & 15 & 96 & 22 & 67 & 335 \\
\hline \multirow[t]{2}{*}{ Todas as áreas } & 2.256 & 1.237 & 218 & 886 & 319 & 959 & 5.875 \\
\hline & $\%$ & $\%$ & $\%$ & $\%$ & $\%$ & $\%$ & $\%$ \\
\hline Ciências da Saúde & 46,6 & 17,3 & 3,9 & 13,0 & 4,9 & 15,0 & 100,0 \\
\hline Ciências Biológicas & 22,1 & 45,1 & 2,2 & 10,0 & 6,4 & 14,1 & 100,0 \\
\hline Ciências Humanas & 35,1 & 4,2 & 8,3 & 23,4 & 5,3 & 23,6 & 100,0 \\
\hline Ciências Sociais Aplicadas & 33,4 & 6,9 & 4,5 & 28,6 & 6,6 & 20,0 & 100,0 \\
\hline Todas as áreas & 38,4 & 21,1 & 3,7 & 15,1 & 5,4 & 16,3 & 100,0 \\
\hline
\end{tabular}

Fonte: CNPq - Diretório dos Grupos de Pesquisa no Brasil, versão 4.1, 2002.

Tabela 10

Linhas de pesquisa relativas ao envelhecimento humano segundo temas selecionados. Brasil, 2000.

\begin{tabular}{lrr}
\hline Temas & N & $\%$ \\
\hline Doenças crônicas e degenerativas. Síndromes geriátricas & 65 & 16,2 \\
Educação. Promoção da saúde. Prevenção de doenças & 44 & 11,0 \\
Envelhecimento biológico & 43 & 10,7 \\
Cuidadores. Domicílio & 29 & 7,2 \\
Saúde mental & 23 & 5,7 \\
Alimentação e nutrição & 21 & 5,2 \\
Epidemiologia & 19 & 4,7 \\
Saúde bucal & 18 & 4,5 \\
Sociedade. História. Cultura & 17 & 4,2 \\
Serviços de saúde. Modelos de atenção & 16 & 4,0 \\
Atividade física & 14 & 3,5 \\
Institucionalização do idoso & 13 & 3,2 \\
Políticas sociais e de saúde & 13 & 3,2 \\
Aposentadoria. Trabalho & 11 & 2,7 \\
Medicamentos. Interações biomateriais & 9 & 2,2 \\
Avaliação multidimensional & 8 & 2,0 \\
Doenças transmissíveis & 7 & 1,7 \\
Recursos humanos & 7 & 1,7 \\
Violência & 6 & 1,5 \\
Dinâmica populacional & 4 & 1,0 \\
Direito. Legislação & 4 & 1,0 \\
Outros & 8 & 2,0 \\
Total & 399 & $\mathbf{1 0 0 , 0}$ \\
\hline
\end{tabular}

Fonte: CNPq - Diretório dos Grupos de Pesquisa no Brasil, versão 4.1, 2002. 
nerativas e das sindromes geriátricas. A educação, a promoção da saúde e a prevenção de doenças, ao lado do envelhecimento biológico, se situam também como temas privilegiados. Um outro amplo leque de temas se apresenta como objetos investigados sob o olhar, por vezes simultâneo, de várias áreas da Ciência.

Estes temas vêm sendo trabalhados por grupos de pesquisa bastante heterogêneos quanto a sua titulação e composição quantitativa de pesquisadores e estudantes.

Em relação aos grupos não específicos, que representam cerca de $70 \%$ do universo estudado, predominam as atividades ligadas as áreas da Saúde e da Biologia, com importante participação das Ciências Humanas. Seus pesquisadores apresentam titulação semelhante às médias nacionais, embora sua capacidade de reprodução da força de trabalho esteja em patamares algo aquém dos padrões que marcam a pesquisa brasileira e a consolidação científica para esses setores corresponda a um ponto a ser alcançado após longa caminhada. Estes, por sua vez, também não são iguais entre si. Um olhar mais individualizado mostra que existem grupos bem situados nos programas de pósgraduação das áreas aos quais estão vinculados, com vários orientandos em diversos níveis de qualificação e vasta produção científica da mais elevada qualidade, inclusive sobre envelhecimento; da mesma forma, há grupos mais frágeis, que têm de construir seu espaço de geração de conhecimento; outros em posição intermediária. Convém reafirmar que, para todos esses, o envelhecimento é um tema, tratado no interior de sua área/subárea previamente estabelecida, seja ela, sociologia, antropologia, demografia, medicina, saúde coletiva, educação física, ou qualquer outra.

E há os grupos especificos, que procuram organizar seus pesquisadores, estudantes e técni- cos em linhas de pesquisa dedicadas exclusivamente às muitas facetas deste complexo processo. No seu conjunto, correspondem a $30 \%$ do universo desta investigação. O olhar das Ciências da Saúde e das Ciências Biológicas é aqui muito mais intenso, materializado, principalmente, por meio da Saúde Coletiva, da Medicina, da Enfermagem e da Odontologia. São grupos criados há bem pouco tempo, particularmente, a partir de 1995. É pequeno o número de grupos vinculados a programas de pósgraduação stricto sensu, mesmo porque estes também se apresentam em número reduzido. A titulação dos pesquisadores líderes destes grupos se encontra aquém dos padrões nacionais, assim como a participação de doutorandos. É possível pensar que a consolidação científica desta área poderá acontecer desde que vinculada a um forte investimento desses pesquisadores e estudantes em sua titulação concomitante a sua associação a programas de pósgraduação que estejam, ou venham a estar, na maior brevidade possível, em condições mais sólidas e estruturadas para produção de conhecimento. O quadro traçado acima corresponde ao perfil geral desses grupos específicos e, evidentemente, não se pode, aqui também, falar em homogeneidade. Há alguns grupos altamente qualificados, com geração de conhecimento reconhecida nacional e/ou internacionalmente, desenvolvendo suas pesquisas em associação com renomados programas de pós-graduação. Como também existem os que dão seus primeiros passos em direção ao domínio dos processos de geração de conhecimento com o raiar do novo milênio; são sanitaristas, enfermeiros, médicos, odontólogos, psicólogos, assistentes sociais, nutricionistas, educadores, entre outros, que se voltam para questões relativas ao envelhecimento, muitas vezes a partir de experiências assistenciais e/ou extensionistas.

\section{Colaboradores}

SD Prado trabalhou na concepção teórica, no levantamento dos dados e na análise do material; e JD Sayd, na concepção teórica e na análise do material. 


\section{Referências bibliográficas}

Conselho Nacional de Desenvolvimento Científico e Tecnológico (CNPq) 2002. Diretório dos Grupos de Pesquisa. Disponível em $<$ http://lattes.cnpq.br/direto rio/>

Conselho Nacional de Desenvolvimento Científico e Tecnológico (CNPq) 2002a. Disponível em $<$ http://lattes. cnpq.br:8888/plataformalattes/index.jsp?menu=diretorio/menu0.htm\&url=diretorio/apresentacao.htm $>$.

Guimarães R, Lourenço R \& Cosac Silvana 2001. A pesquisa em epidemiologia no Brasil. Revista de Saúde Pública. 35(4):321-340. [online, citado 24 agosto 2002]. Disponível em <http://www.scielo.br/scielo. php?script $=$ sci_arttext\&pid $=$ S0034-89102001000 $400001 \& \operatorname{lng}=\mathrm{pt} \& \mathrm{nrm}=\mathrm{iso}>$.
Prado SD \& Tavares EL 1999. Produção acadêmica sobre envelhecimento e velhice: alguns registros preliminares acerca da produção da pós-graduação, pp. 7-9. In SD Prado, EL Tavares \& FRC Silva 1999. Catálogo de teses e dissertações na área do envelhecimento humano. UnATI/CRDE, Rio de Janeiro.

Prado SD \& Lima MA 2000. Catálogo de Cursos de Especialização, Mestrado e Doutorado em geriatria, Gerontologia e Áreas Afins. UnATI/UERJ, Rio de Janeiro.

Prado SD 2004. A pesquisa sobre envelhecimento humano no Brasil: grupos e linhas de pesquisa. Ciência \& Saúde Coletiva 9(1).

Artigo apresentado em 12/6/2003

Aprovado em 15/8/2003

Versão final apresentada em 12/2/2004 